\title{
Secondary Electron-Capture-Decay Isotopes and Implications for the Propagation of Galactic Cosmic Rays
}

\author{
S.M. Niebur ${ }^{1}$, W.R. Binns ${ }^{1}$, E.R. Christian ${ }^{2}$, A.C. Cummings ${ }^{3}$, J.S. George ${ }^{3}$, \\ P.L. Hink ${ }^{1}$, M.H. Israel ${ }^{1}$, J. Klarmann ${ }^{1}$, R.A. Leske ${ }^{3}$, M. Lijowski ${ }^{1}$, \\ R.A. Mewaldt ${ }^{3}$, E.C. Stone ${ }^{3}$, T.T. von Rosenvinge ${ }^{2}$, M.E. Wiedenbeck ${ }^{4}$, and \\ N.E. Yanasak ${ }^{4}$ \\ ${ }^{I}$ Washington University in St. Louis \\ ${ }^{2}$ NASA/Goddard Space Flight Center \\ ${ }^{3}$ California Institute of Technology \\ ${ }^{4}$ Jet Propulsion Laboratory
}

\begin{abstract}
We report the first observation of an energy dependence in the titanium, vanadium, and chromium isotopic abundances in cosmic rays. The observations were made in the $100-500 \mathrm{MeV} /$ nucleon energy interval using data from the Cosmic Ray Isotope Spectrometer on the ACE spacecraft. The ${ }^{51} \mathrm{Cr}$ and ${ }^{49} \mathrm{~V}$ isotopes in cosmic rays are produced by fragmentation of heavier cosmic ray nuclides and decay only by electron capture. The observations indicate that electron-capture decay occurred primarily at the lower energies measured and that there is a resulting energy dependence in the abundances of these isotopes and their decay products.
\end{abstract}

\section{INTRODUCTION}

As galactic cosmic rays propagate through the interstellar medium, they may gain and lose energy as they encounter dense areas of the interstellar medium, supernova remnant shocks, and turbulent magnetic fields. Energy gain due to an encounter with one or more remnant supernova shocks capable of accelerating particles to higher energies has been called distributed acceleration (1). Energy gain due to interaction of cosmic rays with turbulent magnetic fields has been termed diffusive acceleration (2). If cosmic rays undergo either distributed or diffusive acceleration, their energy as they enter the heliosphere could be higher than the energy to which they were initially accelerated.

Certain cosmic-ray isotopes can be used to trace whether the cosmic rays spent much of their lifetime at a lower energy than their energy at heliospheric entry. These isotopes, ${ }^{37} \mathrm{Ar},{ }^{44} \mathrm{Ti},{ }^{49} \mathrm{~V},{ }^{51} \mathrm{Cr},{ }^{55} \mathrm{Fe}$, and ${ }^{57} \mathrm{Co}$, are produced by fragmentation of heavier cosmic rays and decay only by electron capture, which occurs primarily at lower energies where the probability of electron attachment (and thus subsequent decay) is larger. The laboratory half-lives of these isotopes range from 28 days $\left({ }^{51} \mathrm{Cr}\right)$ to 47 years $\left({ }^{44} \mathrm{Ti}\right)$; their half-lives with a single attached electron are approximately twice the laboratory values (3). Since any of these short-lived isotopes would have decayed during the $>10^{5}$ year delay between nucleosynthesis and initial acceleration indicated by $\mathrm{Co}$ and $\mathrm{Ni}$ isotopic ratios (4), these isotopes must have been produced by fragmentation of heavier cosmic rays during galactic propagation. These secondary cosmic rays are produced as bare nuclei that cannot decay by electron capture until they pick up an electron from the medium through which they propagate. At energies above several hundred $\mathrm{MeV} /$ nucleon the probability of electron attachment is low and these isotopes are essentially stable. If evidence of decay were observed at these higher energies, it may be a sign that the nuclei had once propagated at lower energies, where electron attachment and subsequent decay is possible, and thus must have experienced distributed acceleration. The energy dependence of nuclear cross sections, electronattachment cross sections, ionization energy loss, and solar modulation all contribute to energy dependence and must be taken into account before firm conclusions about reacceleration are drawn. 
The energy dependence of electron-capture isotopes and how they can be used to determine the effect of reacceleration has been discussed by Raisbeck et al. (5), Silberberg et al. (1, 6), Letaw et al. (7), and others. These authors describe distributed acceleration and suggest tests, including measurements of secondary electron-capture decay isotopes. In 1998, Soutoul et al. (8) analyzed a combined data set of ISEE-3 and Voyager vanadium isotopic measurements (vanadium isotopes were not clearly resolved in either set) to address this problem with a ${ }^{51} \mathrm{~V} /{ }^{49} \mathrm{~V}$ data point, thereby combining effects of the ${ }^{51} \mathrm{Cr} \rightarrow{ }^{51} \mathrm{~V}$ and ${ }^{49} \mathrm{~V} \rightarrow{ }^{49} \mathrm{Ti}$ decays. The CRIS data set is the first large enough (currently 20 times the number of ISEE$3+$ Voyager vanadium events used by Soutoul et al.) to examine the parent/stable and daughter/stable ratios over a range of energies for both the ${ }^{51} \mathrm{Cr} \rightarrow{ }^{51} \mathrm{~V}$ and ${ }^{49} \mathrm{~V} \rightarrow{ }^{49} \mathrm{Ti}$ decays; CRIS has already collected significant amounts of all isotopes involved. In this paper we will present $\mathrm{Ti}, \mathrm{V}$, and $\mathrm{Cr}$ isotopic measurements that suggest that electron-capture decay has occurred at the lower cosmic-ray energies.

\section{CRIS DATA ANALYSIS}

The Cosmic Ray Isotope Spectrometer (CRIS) on ACE detects cosmic rays with energy $\sim 50-\sim 600$ $\mathrm{MeV} /$ nucleon and charge 2 to 30 (He to $\mathrm{Zn}$ ) with excellent mass resolution (< $0.25 \mathrm{amu})$ and a large collecting power (geometrical factor of $250 \mathrm{~cm}^{2}-\mathrm{sr}$ )(9).
The CRIS instrument consists of a scintillating optical fiber trajectory (SOFT) hodoscope and four silicon solid-state detector telescopes. The SOFT hodoscope, which includes three $x y$ scintillating-fiber planes and a trigger fiber plane, is used to determine trajectories of incident nuclei. The silicon detector telescopes, each a stack of fifteen 3-mm-thick silicon wafers, are used to determine the rate of energy loss and total energy of the detected nuclei.

The CRIS data set contains enough $\mathrm{Ti}, \mathrm{V}$, and $\mathrm{Cr}$ events to be divided into several energy bins over the $100-500 \mathrm{MeV} /$ nucleon energy range (corresponding to interstellar energies between $200-1000$ $\mathrm{MeV} /$ nucleon; the solar modulation parameter $\phi=500$ MV for this time period). After simple data cuts were applied to select a high resolution data set and minimize backgrounds, each of the seven bins we used contains $400-2500$ events, depending on element and energy bin. The energy intervals measured for isotopes of neighboring elements differed by less than $3 \%$.

The resulting histograms each have clear mass separation and an adequate number of particles, as shown in Figure 1. The abundances presented here were obtained by counting the number of particles in each peak, where the peaks are defined as separated at the lowest point of the valley between them. It is estimated that the error in the abundances introduced due to spill-over between adjacent isotopes is approximately $2 \%$, small in comparison to the statistical errors shown in Figures 2, 3, and 4.
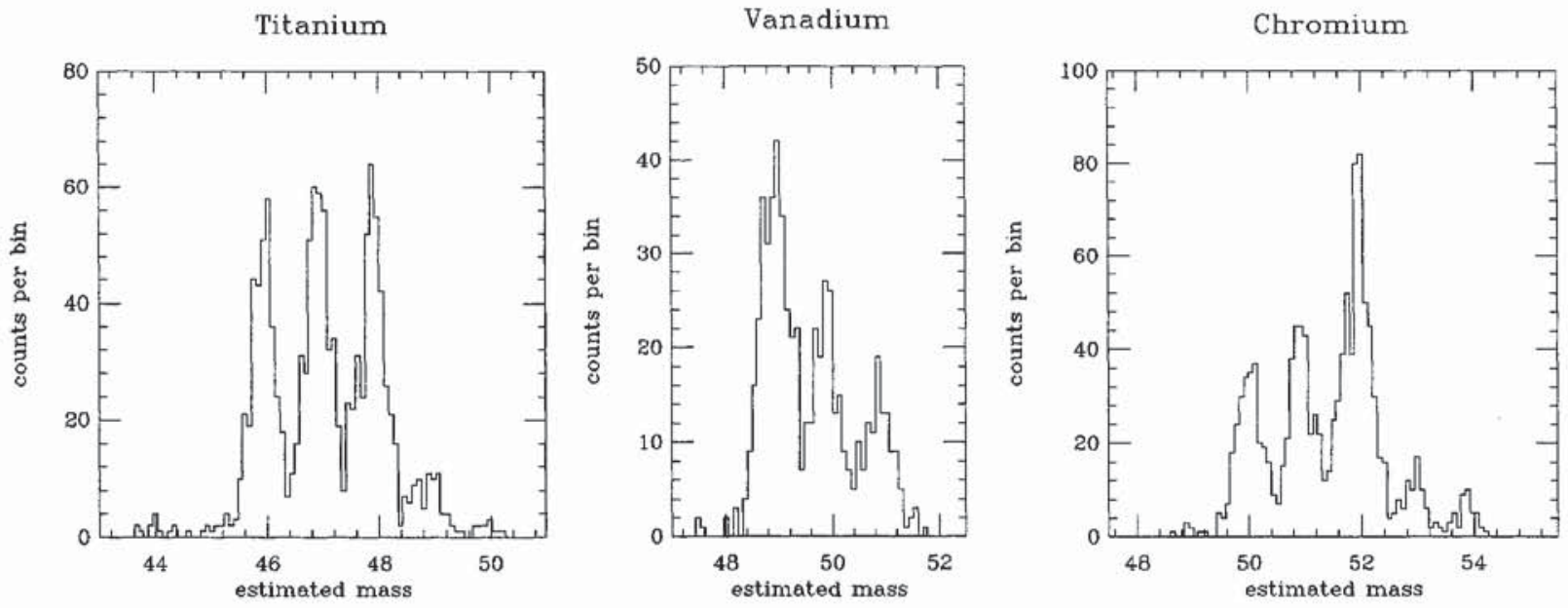

FIGURE 1. Titanium, vanadium, and chromium histograms of CRIS data, energy $300-345 \mathrm{Mev} / \mathrm{nucleon}$, collected during the first 750 days of operation (bin size $=0.1 \mathrm{amu}$ ). The data was divided into seven energy bins; the other 18 histograms also have a mass resolution of $<.25 \mathrm{amu}$. 


\section{RESULTS}

The electron-capture-decay parent abundances, daughter abundances, and parent+daughter abundances relative to the abundances of nearby stable reference isotopes are shown in Figures 2 and $3 .{ }^{52} \mathrm{Cr}$ is used as the stable reference isotope for the ${ }^{51} \mathrm{Cr} \rightarrow{ }^{51} \mathrm{~V}$ decay; the sum of the stable isotopes ${ }^{46} \mathrm{Ti},{ }^{47} \mathrm{Ti}$, and ${ }^{48} \mathrm{Ti}$ is used for the ${ }^{49} \mathrm{~V} \rightarrow{ }^{49} \mathrm{Ti}$ decay. In each case, the daughter isotope abundances increase with decreasing energy, indicating enhanced electron capture and decay at the lower energies. The abundances of the parent isotopes and the sum of the parent and daughter isotopes are consistent with a corresponding depletion in the parent isotopes at lower energies. Nearby stable isotopes do not show these trends; their abundances are not so energy-dependent. Therefore, the ${ }^{51} \mathrm{Cr} \rightarrow{ }^{51} \mathrm{~V}$ and ${ }^{49} \mathrm{~V} \rightarrow{ }^{49} \mathrm{Ti}$ data (particularly the daughter isotopic abundances) indicate that electroncapture decay has occurred more substantially in the lower energy cosmic rays.
These isotopes are compared in Figures 2 and 3 with results from a leaky-box propagation model (described in (10)) that uses energy-dependent nuclear cross sections (11) and solar modulation corrections (12) to produce predictions for observations at $1 \mathrm{AU}$. The dashed line shows the modeling results when electron attachment and subsequent decay are not allowed to occur at any energy. The solid line shows results using the same model but with energydependent electron attachment cross sections (13), allowing electron-capture decay to occur. The two model results converge at higher energies, as expected.

The effect of electron-capture decay would be more pronounced outside the heliosphere, before solar modulation. Adiabatic deceleration in the solar wind stochastically lowers the energy of the cosmic rays, spreading nuclei of a given energy over a range of lower energies $(12,14)$. Therefore, the cosmic-ray nuclei observed at a particular energy propagated through the Galaxy at a range of higher energies.
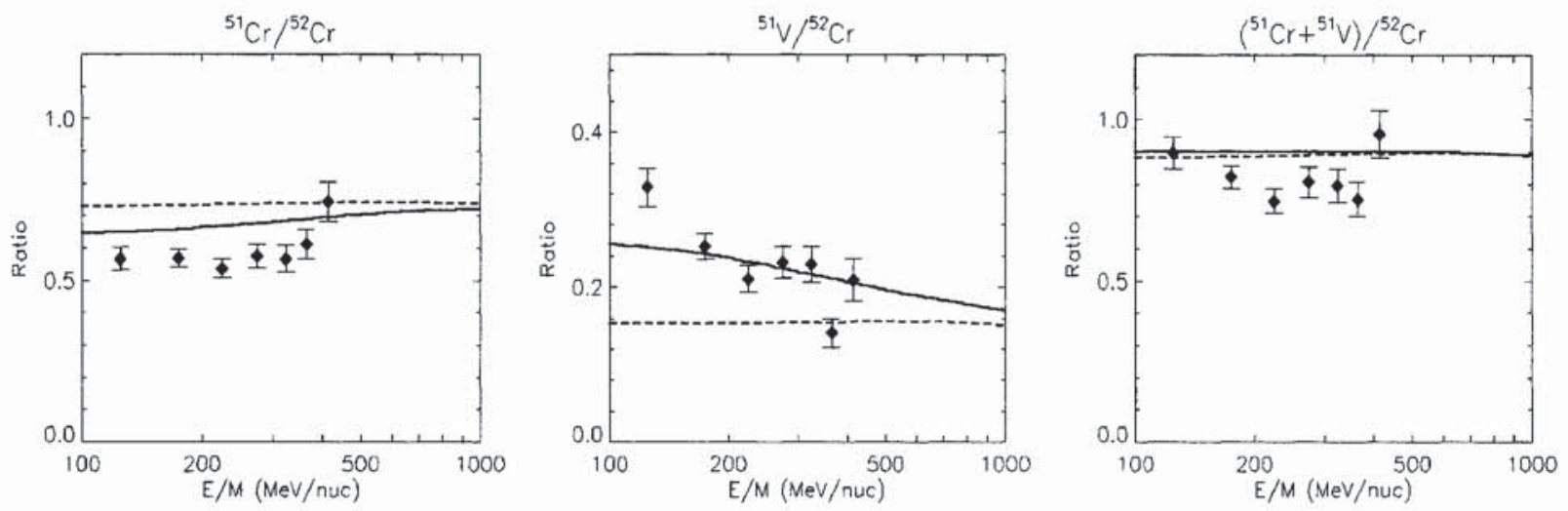

FIGURE 2. Parent and daughter abundances for the ${ }^{51} \mathrm{Cr} \rightarrow{ }^{51} \mathrm{~V}$ decay. Plotted curves are leaky-box propagation model results, with (solid) and without (dashed) electron-capture decay. Reacceleration is not included in this modeling calculation.
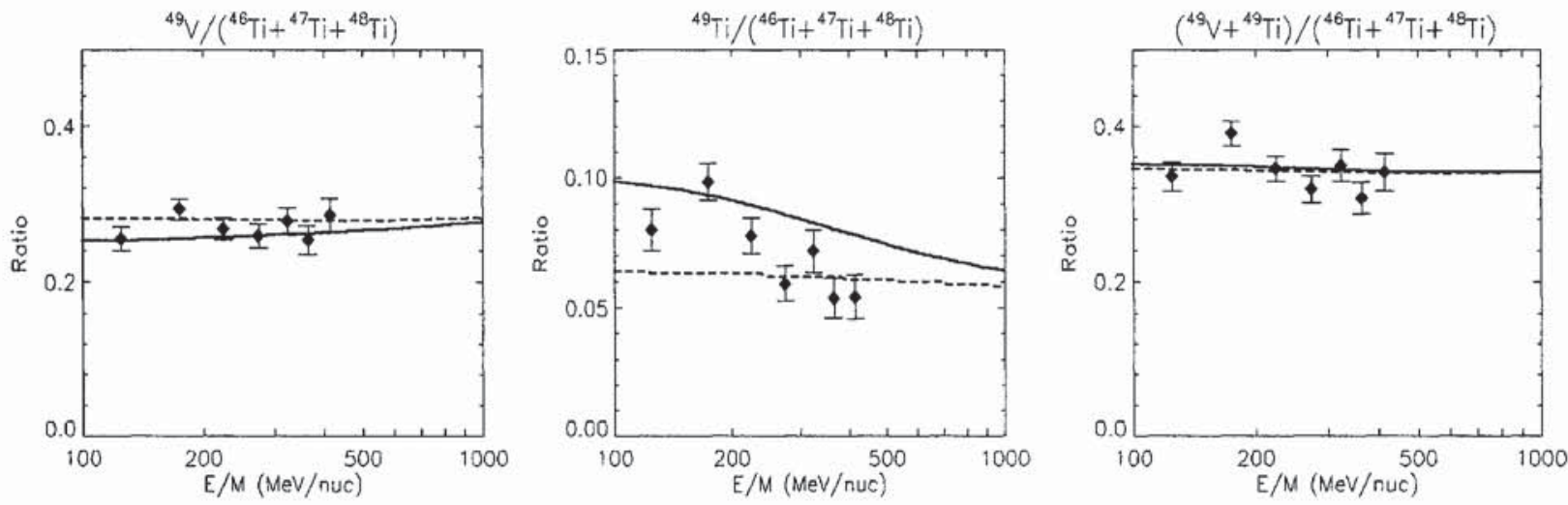

FIGURE 3. Parent and daughter abundances for the ${ }^{49} \mathrm{~V} \rightarrow{ }^{49} \mathrm{Ti}$ decay. Plotted curves are leaky-box propagation model results, with (solid) and without (dashed) electron-capture decay. Reacceleration is not included in this modeling calculation. 

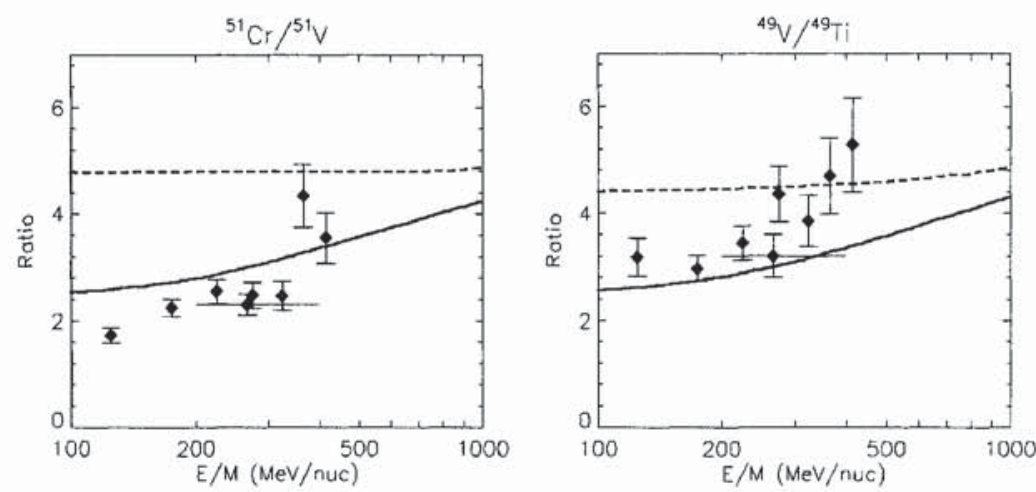

FIGURE 4. Parent/daughter ratios for ${ }^{51} \mathrm{Cr} \rightarrow{ }^{51} \mathrm{~V}$ and ${ }^{49} \mathrm{~V} \rightarrow{ }^{49} \mathrm{Ti}$. Plotted curves are the same as in figures 2 and 3 . The points with horizontal bars show Ulysses results at the energy interval indicated (15).

Figure 4 shows the same data as parent/daughter ratios in order to compare the CRIS data with the recently reported data points of Connell and Simpson (15). The CRIS data in the same energy interval are consistent with the Ulysses data point for each of the two ratios. Although we prefer to examine the parent and daughter energy dependence separately to better isolate effects and check for internal consistency between the observations and the model, we note that these parent/daughter ratios also indicate that electroncapture decay has occurred more often at the lower energies.

\section{DISCUSSION AND CONCLUSION}

This preliminary data analysis indicates that electron capture and decay have occurred primarily at the lower cosmic-ray energies. These data are in qualitative agreement with our leaky-box propagation model that includes energy-dependent electron attachment cross sections (solid line in figures 2, 3, and 4), but does not include reacceleration during transport. The offset observed in several ratios may be due to errors in the nuclear cross sections.

We plan to incorporate reacceleration into our model and compare our data on the secondary electron-capture decay isotopes $\left({ }^{37} \mathrm{Ar},{ }^{44} \mathrm{Ti},{ }^{49} \mathrm{~V},{ }^{51} \mathrm{Cr}\right.$, ${ }^{55} \mathrm{Fe}$, and ${ }^{57} \mathrm{Co}$ ) and their decay products with this and other models in order to quantify the amount of acceleration that could have occurred during cosmic ray propagation through the interstellar medium. As CRIS continues to collect data, the statistical uncertainties will be reduced, allowing more stringent comparisons with various model results. We may also be able to use these data to better evaluate the effects of solar modulation.

\section{ACKNOWLEDGMENT}

This research was supported by the National Aeronautics and Space Administration at the California Institute of Technology (under grant NAG5-6912), the Goddard Space Flight Center, the Jet Propulsion Laboratory, and Washington University.

\section{REFERENCES}

1. Silberberg, R. et al., Phys. Rev. Lett. 51, 1217-1220 (1983).

2. Heinbach, U., and Simon, M., Astrophys. J. 441, 209221 (1995).

3. Wilson, L. W. Ph.D. thesis. University of California at Berkeley. Lawrence Berkeley Laboratory Report LBL7723 (1978).

4. Wiedenbeck, M. E. et al., Astrophys. J. 523, L61-L64 (1999).

5. Raisbeck, G. M. et al., Proc. $14^{\text {th }}$ Internat. Cosmic Ray Conf. (Munich) 2, 560-563 (1975).

6. Silberberg, R., and Tsao, C. H., Phys. Reports 191(6), 351-408 (1990).

7. Letaw, J. R., Silberberg, R., and Tsao, C. H., Astrophys. J. Supp. 56, 369-391 (1984).

8. Soutoul, A. et al., Astron. Astrophys. 336, L61-L64 (1998).

9. Stone, E. C. et al., Space Sci. Rev. 88(1), 285-356 (1998).

10. Leske, R. A., Astrophys. J. 405, 567-583 (1993).

11. Webber, W. R. et al., Phys. Rev. C. 58(6), 3539-3552 (1998).

12. Goldstein, M.L., Fisk, L. A., and Ramaty, R. Phys. Rev. Lett. 25, 832-835 (1970).

13. Crawford, H. J. Ph.D. Thesis. University of California at Berkeley. Lawrence Berkeley Laboratory Report LBL-8807 (1979).

14. Labrador, A. W., and Mewaldt, R. A., Astrophys. J. 480, 371-376 (1997).

15. Connell, J. J., and Simpson, J. A., Proc. $26^{\text {th }}$ Internat. Cosmic Ray Conf. (Salt Lake City) 3, 33-36 (1999). 